\title{
Progress in stellarator research at IPP-Kharkov
}

Vladimir E. Moiseenko, Oleksiy V. Lozin,

Anatoliy M. Shapoval,

Mykola B. Dreval, Yuliya S. Kulyk,

Yuri K. Mironov, Vladyslav S. Romanov, Valeriy K. Pashnev, Eduard L. Sorokovoy, Andriy A. Petrushenya, Fedir I. Ozherel'ev, Mykhailo M. Kozulya, Volodymyr G. Konovalov, Sergiy M. Maznichenko, Igor E. Garkusha

\begin{abstract}
A new antenna of 'crankshaft' type has been installed in the Uragan-2M device in order to increase the plasma density and heating below the ion cyclotron frequency. Antenna operation is modelled by 1D code, which solves boundary problem for time-harmonic Maxwell's equations in radially non-uniform plasma cylinder. In recent experiments with this antenna, the SXR, CV, OV and OII emission measurements indicate that the light impurity radiation barrier is overcame at this device. Plasma with a temperature of $\sim 50 \mathrm{eV}$ exists during a short period of a few milliseconds. Then the radiation collapse comes owing to strong influx of impurities to the plasma column. A new magnetic diagnostics has been installed at Uragan-3M. Using it the poloidal magnetic field is measured and the shift of toroidal current in major radius is registered. A miniature pinhole camera array for spatially and temporally resolved measurements of soft X-ray (SXR) plasma emission has been recently installed on the U-3M. Different shapes of the SXR emission profile has been observed in different discharge conditions.
\end{abstract}

Key words: stellarator $\bullet$ radio-frequency heating $\bullet$ low frequency modes $\bullet$ magnetic diagnostics

V. E. Moiseenko ${ }^{\varpi}$, O. V. Lozin, A. M. Shapoval, M. B. Dreval, Yu. S. Kulyk, Yu. K. Mironov, V. S. Romanov, V. K. Pashnev, E. L. Sorokovoy, A. A. Petrushenya, F. I. Ozherel'ev, M. M. Kozulya, V. G. Konovalov, S. M. Maznichenko, I. E. Garkusha National Science Center 'Kharkiv Institute of Physics and Technology',

1 Akademichna Str., Kharkiv 61108, Ukraine, Tel.: +38 057335 6650, Fax: +38 057335 2664,

E-mail: moiseenk@ipp.kharkov.ua

Received: 25 September 2015

Accepted: 3 December 2015

\section{Introduction}

There are two stellarator-type devices at the Institute of Plasma Physics, National Science Center 'Kharkiv Institute of Physics and Technology'. The bigger one is the Uragan-2M (U-2M) facility [1] that is a middle-size $l=2$ torsatron machine with $m=4$ helical field periods and additional toroidal field coils. The torus major radius is $R_{0}=1.7 \mathrm{~m}$, an average last-closed magnetic-surface radius is $a=0.22 \mathrm{~m}$. The magnetic field does not exceed $B=0.9 \mathrm{~T}$ at present time because of power supply limitations. In addition to the helical winding, it has coils for toroidal magnetic field, which are fed separately. This ensures some flexibility in a control of the magnetic configuration.

The smaller Uragan-3M (U-3M) device is also a torsatron, but with $l=3$ and $m=9$. In this machine, the torus major radius is $R_{0}=1 \mathrm{~m}$, an average last-closed magnetic-surface radius is $a=0.12 \mathrm{~m}$ and the magnetic field ranges up to $1 \mathrm{~T}$. The distinctive feature of this device is the magnetic configuration with a natural divertor. Both machines operate sequentially, each after the other.

In this paper, the recent results from both machines are presented and discussed. In the next section, a new 'crankshaft' antenna is presented and the experiments with RF plasma production and heating in the Uragan-2M are reported and discussed. Section "Dynamics of longitudinal plasma current during RF plasma heating in Uragan-3M" describes a new magnetic diagnostics at the Uragan-3M and 

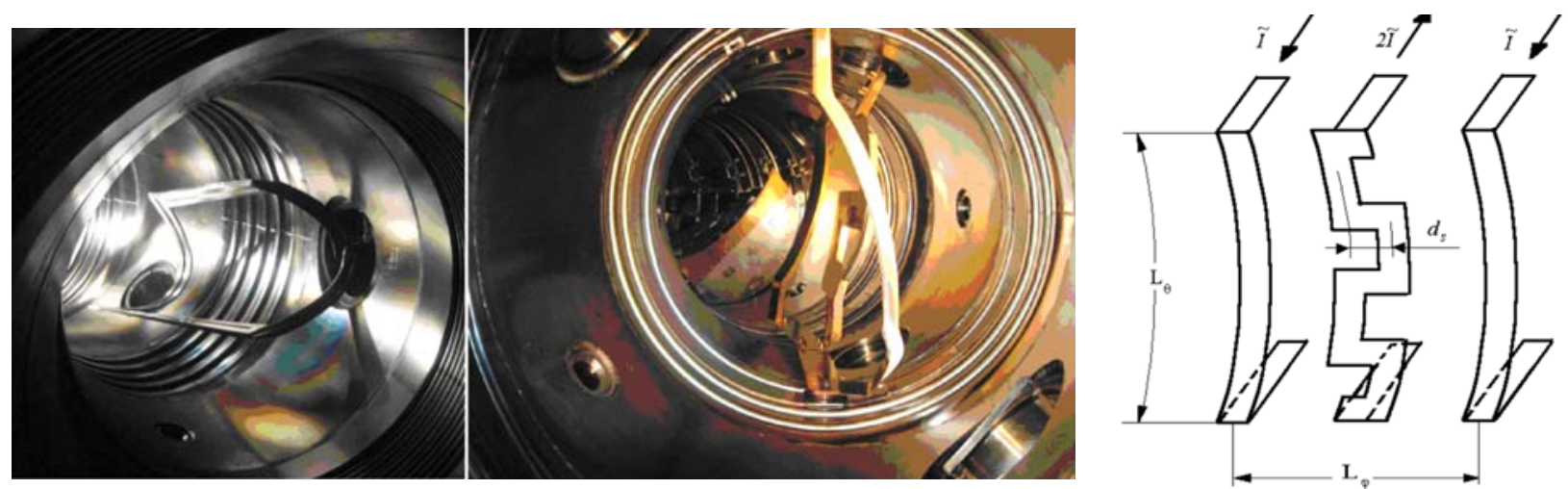

Fig. 1. Frame (left) and crankshaft (centre) antennas in the Uragan-2M vacuum vessel. The crankshaft antenna sketch (right).

its first results. In section "SXR data from Uragan-3M", the studies with a soft X-ray miniature pinhole camera are summarized. The paper is finalised by the last section.

\section{Plasma production and heating in Uragan-2M device with 'crankshaft' antenna}

Present Uragan-2M torsatron experiments suffer still from an accidental vacuum oil injection that occurred few years ago. After this, strong efforts have been applied to remove the oil using RF discharges and baking. During the recent period, the device was used for studies, which do not require hot dense plasmas, such as studies of the radio-frequency (RF) wall conditioning [2], runaway electron measurements, etc. Each campaign of the RF plasma production and heating was attempted to improve plasma parameters. Plasma could be created at frequencies below, but quite close to the ion cyclotron. The plasma density was about $n_{\mathrm{e}} \sim 10^{12} \mathrm{~cm}^{-3}$. Plasma temperature was estimated as a relatively low one, because the CIII line emission dominated in the recorded optical spectrum. In recent experiments we have obtained more optimistic results.

The RF plasma production and heating in Uragan stellarators is traditionally performed near or below the ion cyclotron frequency. In this range of frequencies two waves may propagate: the fast wave (FW) and the slow wave (SW). The fast wave has two branches, the fast magnetosonic wave (FMSW) with a longer longitudinal wavelength and the fast Alfven wave (FAW), which is shorter. The FMSW propagates in medium-size and big machines. In single-ion component plasma, its damping is small, both ion cyclotron and Landau waves. The FAW can propagate in smaller machines, but it is hard to couple it if it is single one (i.e. without presence of the Alfven resonance), and its damping is also not sufficient. The SW has a short wavelength, and its damping via Landau mechanism is high. Below the ion cyclotron frequency, the FAW converts into the SW and vice versa at the Alfven resonance layer [3, 4]. This conversion is a basis for the Alfven resonance heating (ARH) scenario. Within it, the FAW wave field is excited by an antenna. Then the field penetrates into plasma though the cut-off region, reaches the Alfven resonance layer and conversion to the SW occurs. After that the SW is damped on plasma electrons. Such complex scenario allows one to avoid the SW damping at a plasma periphery.

An ARH scenario with compact antennas $[4,5]$ is practiced at both Uragan devices. The antennas used are, in principle, similar to those used for ion cyclotron heating, with the difference that they have, preferably, more concentrated $k_{\|}$spectrum. A crankshaft [6] antenna (see Fig. 1) has been designed, manufactured and installed at the Uragan-2M. It has three $\pi$-phased straps, and the central strap is fed with double current. The antenna is a version of three half-turn (THT) antenna and differs from that one by wiggled central strap. The wiggling increases antenna ability to excite the SW, that is favourable for the plasma production. The antenna operation is modelled by a 1D RF-code, which solves boundary problem for time-harmonic Maxwell's equations in a radially non-uniform plasma cylinder. The modelling was used to choose antenna dimensions and the operational regime. An example of such calculation series is displayed in Figs. 2 and 3.

In the described calculations, the plasma density profile is chosen to be a parabolic one with a

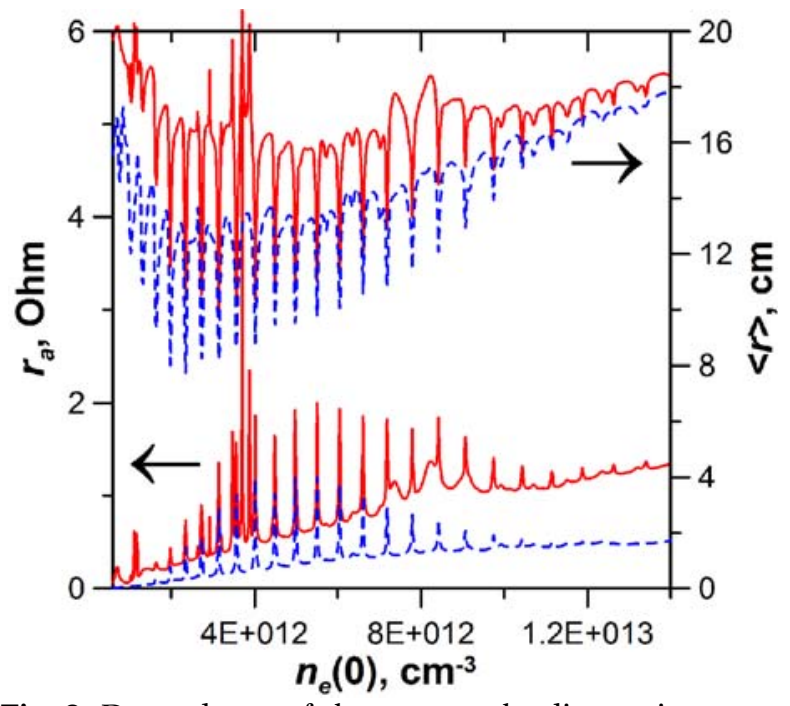

Fig. 2. Dependence of the antenna loading resistance $r_{a}$ and an average radius of the power deposition $<r>$ on the central plasma density $n_{\mathrm{e}}(0)$. Solid curves are for the crankshaft antenna and dashed curves are for the THT antenna. 


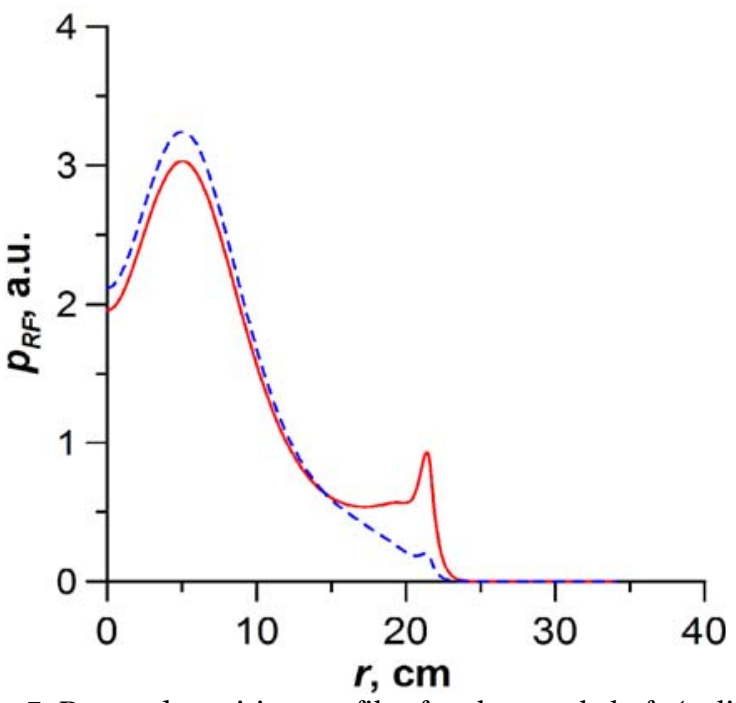

Fig. 3. Power deposition profiles for the crankshaft (solid curve) and THT (dashed curve) antennas.

plasma radius $a=22 \mathrm{~cm}$. The electron temperature decreases from $T_{\mathrm{e}}(0)=100 \mathrm{eV}$ to $T_{\mathrm{e}}(a)=50 \mathrm{eV}$ at the plasma edge. The magnetic field is $B_{0}=3.7 \mathrm{kG}$ and the RF frequency is $f_{\mathrm{RF}}=3.9 \mathrm{MHz}$.

Figure 2 displays the dependence of the antenna loading resistance $r_{a}=2 P_{\mathrm{RF}} / I_{c s}^{2}$ and the average radius of power deposition

$$
<r>=\frac{\int_{0}^{a} p_{\mathrm{RF}} r^{2} \mathrm{~d} r}{\int_{0}^{a} p_{\mathrm{RF}} r \mathrm{~d} r}
$$

where $P_{\mathrm{RF}}$ is the RF power dissipated in plasma, $I_{c s}$ is the RF current in the central strap and $p_{\mathrm{RF}}$ is the RF power density. Two calculations are displayed, with and without 'crankshaft' wiggling. The antenna loading resistance is higher for the crankshaft antenna than for the THT antenna, especially at low plasma densities. It increases with a plasma density for both antennas. The characteristic peaks are a result of the global resonances of the FAW [7]. They are narrow since this wave is weakly damped. An average radius of the power deposition also reacts on them by drop in its value. This occurs because this wave is centrally located. The power deposition is worse for the crankshaft antenna. The optimum of the power deposition is observed at the plasma density $n_{\mathrm{e}}(0) \approx$ $5 \times 10^{12} \mathrm{~cm}^{-3}$, while for the THT antenna the optimum density is lower and amount to $n_{\mathrm{e}}(0) \approx 3 \times 10^{12} \mathrm{~cm}^{-3}$. As regards the power deposition profiles at the density value $n_{\mathrm{e}}(0) \approx 5 \times 10^{12} \mathrm{~cm}^{-3}$ (shown in Fig. 3), they are similar in a plasma core for both antennas, and the crankshaft antenna has some power deposition also at a plasma periphery.

In the experiments, the frame antenna (shown in Fig. 1) starts first. The heating frequency is $f=$ $5.35 \mathrm{MHz}$ and the power is below $50 \mathrm{~kW}$. It creates cold plasma with the density $n_{\mathrm{e}} \sim 10^{11} \mathrm{~cm}^{-3}$. Then the crankshaft antenna is switched on with a frequency $f=4.9 \mathrm{MHz}$ and more powerful pulse $P_{\mathrm{RF}} \sim 150 \mathrm{~kW}$. The RF frequency of the crankshaft antenna is at the experimental optimum and higher than predicted by the numerical modelling. This could be explained by

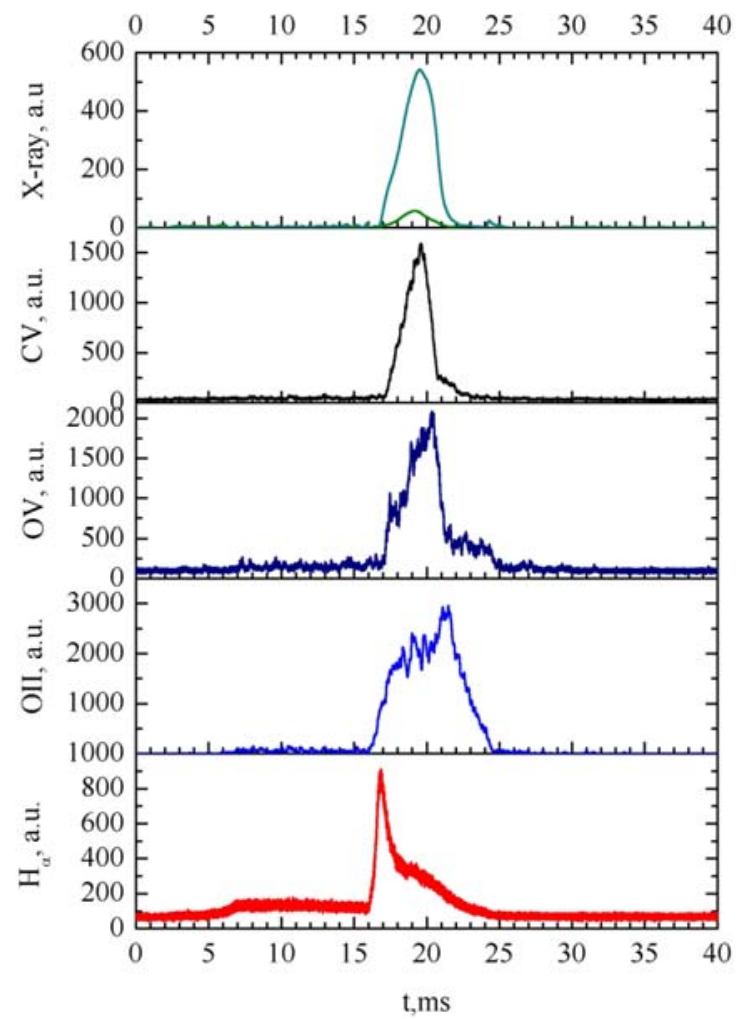

Fig. 4. Time dependence of the SXR and optical emissions (a.u.).

an influence of impurities on the plasma dielectric properties. This influence is not accounted for in the numerical model. The discharge parameters are as follows: the magnetic field had a value $B_{0}=0.37 \mathrm{~T}$, hydrogen gas pressure was $p_{\mathrm{H} 2}=1.8 \times 10^{-3} \mathrm{~Pa}$. During this experimental campaign, the plasma density was measured with the single-chord interferometer, $\mathrm{H}_{\alpha}$, OII, OV and CV optical line emissions and double-foil soft X-ray (SXR) emission measurements are made. The plasma density quickly increases to the value of $n_{\mathrm{e}} \approx 2.5 \times 10^{12} \mathrm{~cm}^{-3}$ and then slowly ramps up to $n_{\mathrm{e}} \approx 3.5 \times 10^{12} \mathrm{~cm}^{-3}$. The $\mathrm{H}_{\alpha}$ strong peak indicates the neutral gas burn-out and fully ionised plasma formation (see Fig. 4)

The OII emission appears after $\mathrm{H}_{\alpha}$ peak, rises up first quickly and then slowly. When the OII rise slows down the $\mathrm{OV}$ and $\mathrm{CV}$ emission consecutively appear indicating the electron plasma heating up to the temperature $T_{\mathrm{e}} \geq 100 \mathrm{eV}$. The average plasma temperature, as determined by the SXR diagnostics, reaches the value $T_{\mathrm{e}} \approx 50 \mathrm{eV}$. Since the instant $t=19.5 \mathrm{~ms}$, a degradation of the discharge begins. Then the OII emission starts to increase, and the $\mathrm{CV}$ emission decreases. The OV emission starts to decrease a bit later. All these observations together with a SXR temperature decrease, indicating the poisoning of the discharge with impurities and turning it to the weak state.

\section{Dynamics of longitudinal plasma current during RF plasma heating in Uragan-3M}

Recently a set of 15 magnetic probes (so-called Mirnov coils) have been installed at a minor radius 


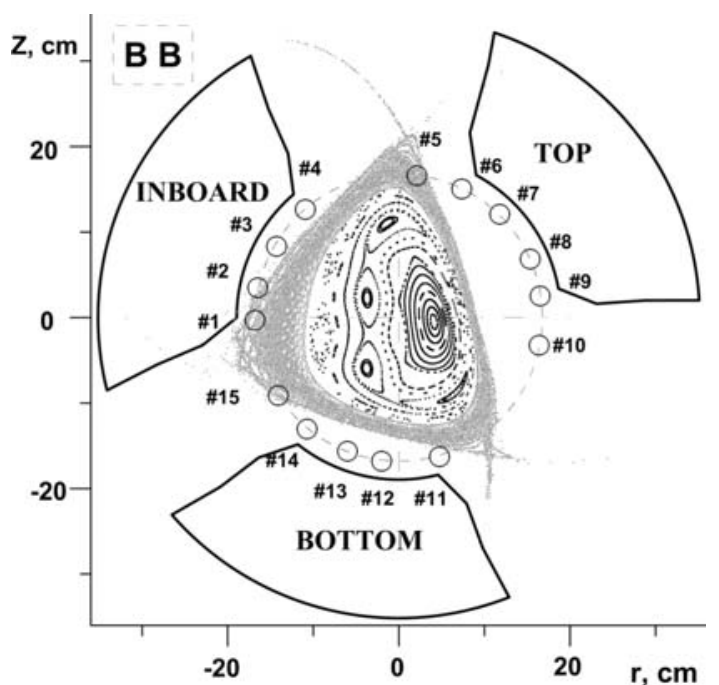

Fig. 5. Poloidal cross-section of the torus showing positions of the helical coils, magnetic sensors and vacuum magnetic surfaces.

$r_{p}=16.8 \mathrm{~cm}$ in one poloidal cross-section of the U-3M torus, and preliminary results on the toroidal plasma current and angular distributions of current-produced poloidal component of a magnetic field were obtained. Locations of the probes and positions of the cross-sectional view of helical coils are shown in Fig. 5. There are also shown the calculated magnetic surfaces (the Poincaré magnetic field line traces) in this torus cross-section.

As it can be seen, the magnetic axis with the most inner magnetic surfaces is shifted outward by $\approx+4 \mathrm{~cm}$ relatively to the geometrical axis. At the same time, the 'centres' of more exterior magnetic surfaces are located nearby the geometrical axis.

The probe system gives us a possibility to calculate a shift of the centres of poloidal magnetic field distributions of the plasma currents in the horizontal direction. The calculations can be carried out according to the formula:

$$
\text { (2) } \begin{gathered}
\Delta=-r_{p} \cdot\left[U_{1}-0.5\left(U_{9}+U_{10}\right)\right] /\left[U_{1}+0.5\left(U_{9}\right.\right. \\
\left.\left.+U_{10}\right)\right]
\end{gathered}
$$

where $U_{1}, U_{9}$ and $U_{10}$ are the signals from the sensors with corresponding numbers shown in Fig. 5; $r_{p}$ is the minor radius, where magnetic probes are installed.

Some preliminary results of such calculations are presented in Fig. 6 as the poloidal distributions of magnetic field generated by the toroidal current, and in Fig. 7 as time dependence of the horizontal shift of these distributions. The measurements were performed in low-density plasma regimes $\left(n_{\mathrm{e}} \leq 2 \times\right.$ $10^{12} \mathrm{~cm}^{-3}, B_{\varphi} \approx 0.72 \mathrm{~T}$ ), when the toroidal plasma current was within the range $\leq 2 \mathrm{kA}$ [8]. The results are given for time beginning $30 \mathrm{~ms}$ after the RF discharge start.

At the initial stage of the discharge, $t=30 \mathrm{~ms}$ (see Fig. 6, black line, and Fig. 7a), the distribution is shifted outwards from the geometrical torus centre, $\Delta \approx+4 \mathrm{~cm}$. As Fig. 7 a shows, later in time, the $\Delta$ value decreases by $2.4 \mathrm{~cm}$ at $t=35 \mathrm{~ms}$ and by $0.6 \mathrm{~cm}$ at $t=52 \mathrm{~ms}$. Afterward (for $t>52 \mathrm{~ms}$ ), it

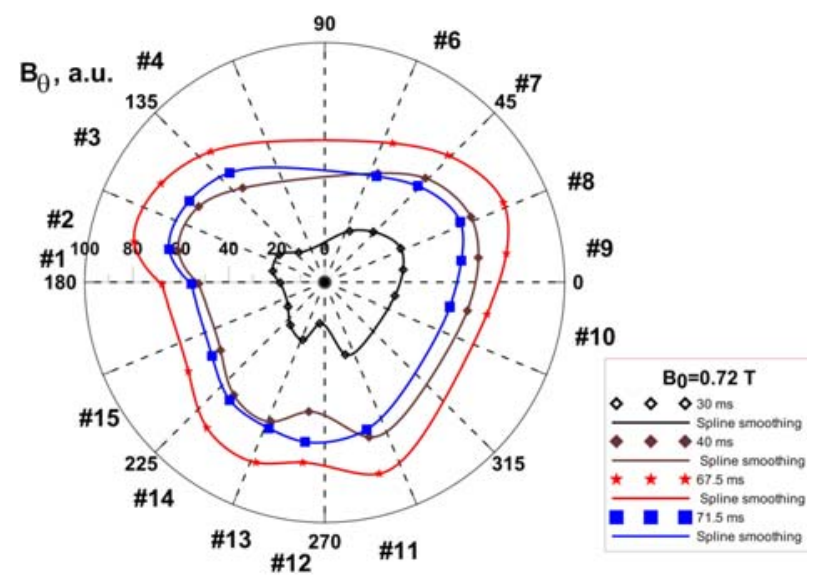

Fig. 6. Distribution in polar coordinates of the magnetic field produced by toroidal current for four different instants $t$.

stays almost unchanged up to the RF pulse end, when $\Delta$ becomes negative $(-0.7 \mathrm{~cm})$ at $t=72 \mathrm{~ms}$, that is, 2 ms after the RF power switch off. The initial (at $t=30 \mathrm{~ms}$ ) and final (at $t=72 \mathrm{~ms}$ ) $\Delta$ values correspond to the location of the magnetic axis (with internal magnetic surfaces) and the centre of external magnetic surfaces for the vacuum magnetic configuration, that is, $\approx+4 \mathrm{~cm}$ and $\approx-1 \mathrm{~cm}$ (see Fig. 6). Such correspondence can be considered as an indication on a big difference between radial distributions of the induced toroidal current at the initial stage of the discharge $(t \approx 30 \mathrm{~ms})$ and at later time intervals, down to discharge afterglow (at $t \geq 72 \mathrm{~ms}$ ).

Figure $7 \mathrm{~b}$ shows the dynamics of $\Delta$ near the time of a transition into the mode of the improved confinement $\approx 35 \mathrm{~ms}$. It is clear that the transition is followed by some increase of $\Delta$, which means that the current is shifted to the magnetic axis, that is, to a more picked profile of plasma current realised at the time of the transition.

\section{SXR data from Uragan-3M}

A 2- $\mu$ m-thick $\mathrm{Al}$ foil has been installed in the U-3M SXR pinhole diagnostics to cut off a low-energy

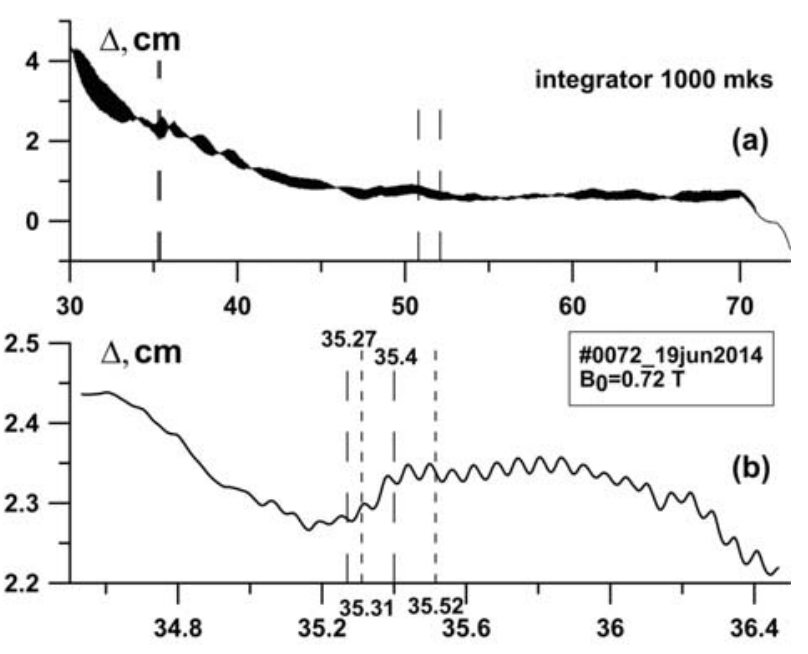

Fig. 7. Shift of the centers of poloidal magnetic field distributions. 


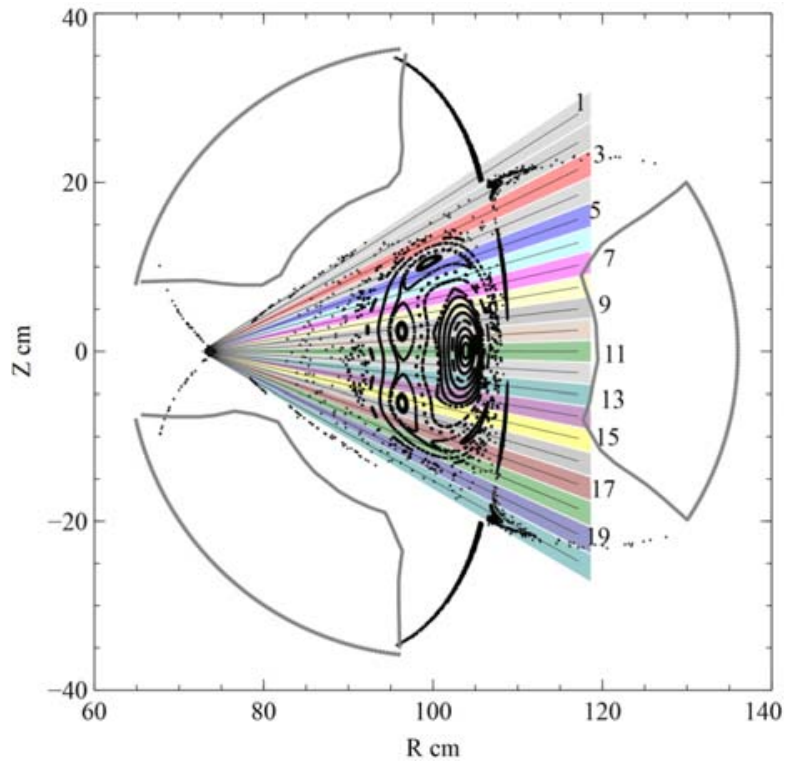

Fig. 8. Lines of sight and viewing angles of the SXR detector array across the magnetic surfaces of the U-3M cross-section 'A-A'.

radiation. The SXR camera consists of a 20-channel photodiode linear-array of the IRD AXUV-20EL type. The camera is viewing horizontally through a symmetric plasma cross-section 'A-A', as shown in Fig. 8.

The SXR photodiode photocurrent amplifiers gain is $2.5 \times 10^{7} \mathrm{~V} / \mathrm{A}$. The amplifiers bandwidth is $10 \mathrm{kHz}$. It allows to suppress successfully high level RF noise and to record SXR signals [9]

\section{SXR profile behaviour during the transition}

The transition is observed in the low-density frame-antenna U-3M discharges [10, 11]. Figure 9a shows SXR signals from channels with opposite impact parameters. Figure $9 \mathrm{~b}$ shows clear $20-\mathrm{kHz}$ magnetohydrodynamics (MHD) activity, which is always accompanied by the transition [9]. As it can be seen from Fig. 9, the transition is related to a complex U-3M plasma column perturbation associated with the MHD activity. The SXR profile vertical shift is
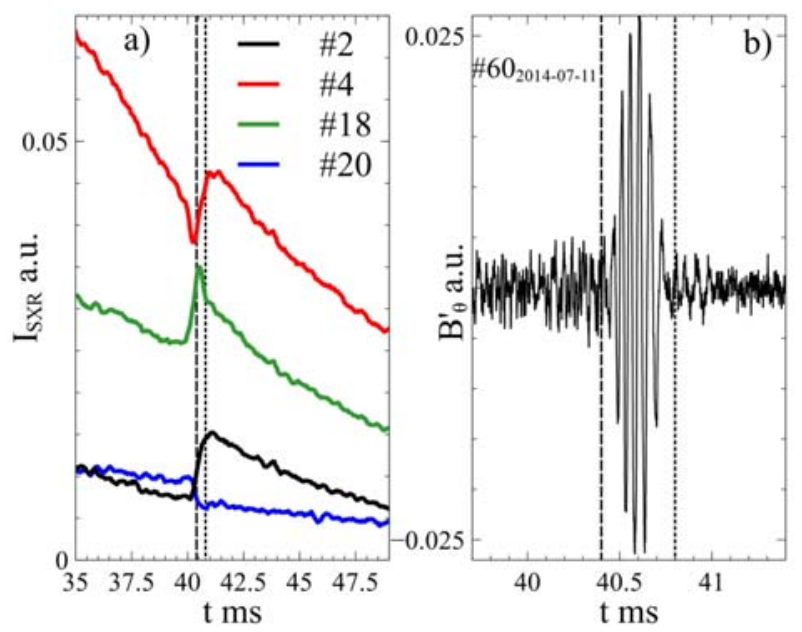

Fig. 9. Evolution of SXR signal in channels with opposite impact parameters (a) and magnetic probe data (b).

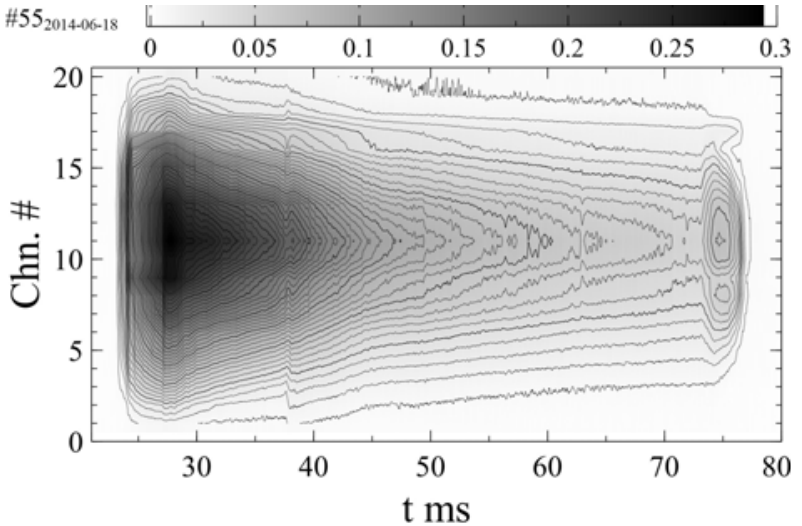

Fig. 10. SXR profile in a frame-antenna discharge.

observed at the plasma periphery. Therefore, the signal from channel \#20 is dropping and the signal from channel \#2 is rising. A significant phase delay in the SXR emission perturbation is observed in deeper channels \#4 and \#18. This experimental effect can be explained by a rotation of some plasma perturbations.

Detailed SXR-profile temporal behaviour in a discharge with the transition is shown in Fig. 10. In addition to the complex plasma column movement, a transient decrease of the SXR emission decay is also observed. Rather small SXR emission rise is not clear enough for the SXR emission gradient steepening localisation. At present, it is impossible to separate the MHD event and possible confinement improvement influence on the SXR emission. We should note that, due to the SXR electronics bandwidth limitation, the $20 \mathrm{kHz}$ mode is not observed in the SXR diagnostics.

An MHD phenomenon, previously treated as a transition to H-like mode, is observed by the SXR diagnostic array as well as magnetic probes. We are considering this transition as an MHD burst.

\section{SXR profiles in various Uragan-3M discharges}

A substantial SXR emission rise after the RF power switching off is always observed in the low-density frame-antenna discharges [9]. An example of this increase is shown in Fig. 10 at $t=73-75 \mathrm{~ms}$. The SXR signal increase is evidently associated with the plasma density increase, which is always observed at the end of this type of the U-3M discharge [11]. An asymmetry of the SXR emission profile is clearly shown in a normalised SXR profile (see Fig. 11) at the break-down/build-up stage. This asymmetry can be associated with a transient modification of the RF power deposition in the break-down stage. A peaking of the normalised SXR emission profile after the RF power turning off (at 73-75 ms) does evidently represent better plasma confinement in a central region, but rather some influence of the edge RF power deposition. This deposition disappears after the discharge switching off. The SXR emission from the periphery is dropping due to worst confinement in this region. 

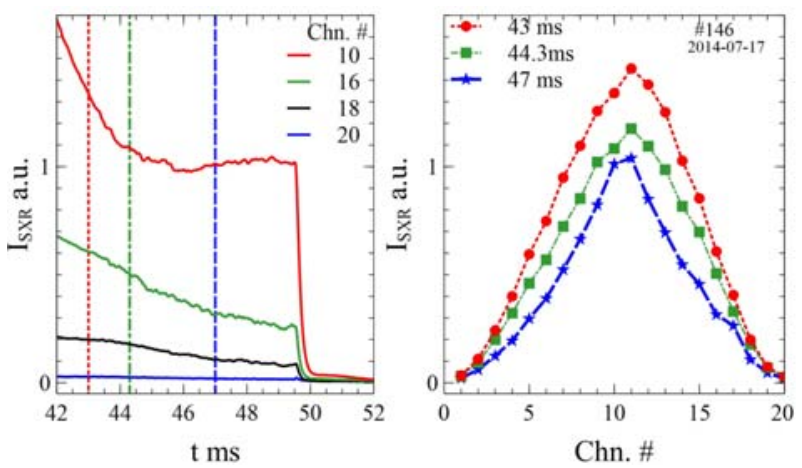

Fig. 11. SXR profile at the end of a THT discharge.

A substantial amount of the SXR data in the three half-turn (THT) antenna discharges [9] has also been obtained recently in the U-3M machine. An example of the SXR profile modification in the final degradation stage of the THT antenna discharge is shown in Fig. 11.

A clear peaking of the SXR emission profile near the end of discharge indicates that some plasma cooling at the periphery occurs. Probably, a degradation of the RF power absorption at the periphery appears at the end of the THT-antenna discharge under consideration.

Another example of the THT-antenna discharge is shown in Fig. 12. The first peak (at 12-16 ms) corresponds to the low-power-frame-antenna plasma creation stage. The second stage represents medium power THT-antenna discharge.

\section{Summary}

A new antenna of 'crankshaft' type has been installed in the Uragan-2M device in order to achieve a plasma density increase and heating below the ion cyclotron frequency. The antenna operation has been modelled with a 1D-code, which solves boundary problem for time-harmonic Maxwell's equations in a radially non-uniform plasma cylinder. In recent experiments with this antenna, the SXR, CV, OV and OII emission measurements have indicated that the light impurity radiation barrier is overcame at this device. Plasma with a temperature of $\sim 50 \mathrm{eV}$ exists during a short period of a few milliseconds. Then the radiation collapse appears owing to strong influx of impurities to the plasma column.

A new magnetic diagnostics has been installed also at the Uragan-3M machine. Using it the poloidal magnetic field has been measured and the shift of toroidal current in major radius has been recorded. It has been observed that after a transition into the mode of an improved confinement more picked profiles of plasma current are formed.

A miniature pinhole camera array for spatially and temporally resolved measurements of the soft $\mathrm{X}$-ray plasma emission has recently been installed on the U-3M. Different shapes of the SXR emission profile have been observed at various discharge conditions. Different types of the low-frequency $(f \approx 1 \mathrm{kHz})$ SXR signals fluctuations have been

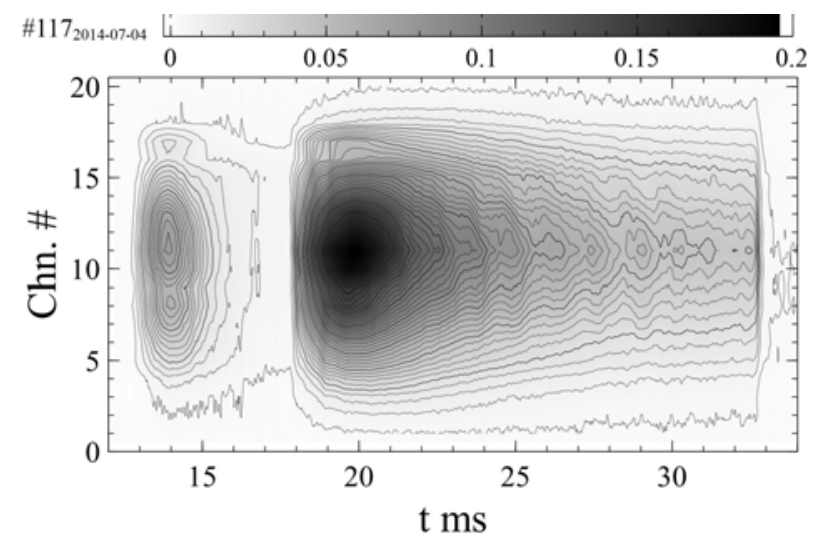

Fig. 12. SXR profile in a THT-antenna discharge with the frame-antenna plasma build-up.

observed in the U-3M torsatron. An MHD phenomenon, previously treated as a transition to an $\mathrm{H}$-like mode, has been observed with the SXR diagnostic array as well as magnetic probes. We are considering this transition as an MHD burst.

This work was performed at the NSC KIPT in Kharkov, Ukraine.

\section{References}

1. Tereshin, V. I., Beletskii, A. A., Berezhnyj, V. L., Burchenko, P. Ya., Chechkin, V. V., Grigor'eva, L. I., Gubarev, S. P., Konovalov, V. G., Kulaga, A. E., Kurilo, D. V., Lesnyakov, G. G., Losin, A. V., Maznichenko, S. M., Mironov, Yu. K., Moiseenko, V. E., Ozherel'ev, F. I., Opaleva, G. P., Pashnev, V. K., Pavlichenko, O. S., Prokopenko, A. V., Romanov, V. S., Sergeev, Zu. F., Skibenko, A. I., Shapoval, A. N., Shtan', A. F., Shvets, O. M., Solodovchenko, S. I., Sorokovoy, Ze. L., Tashi, V. S., Tarasov, I. K., Tsybenko, S. A., Voitsenya, V. S., Volkov, E. D., \& Zolototrubova, M. I. (2008). First results of the renewed Uragan-2M torsatron. In Proceedings of the 35th EPS Conference on Plasma Physics, 9-13 June 2008. Hersonissos, Greece. ECA 32D:P-1.061.

2. Moiseenko, V. E., Lozin, A. V., Chechkin, V. V., Chernyshenko, V. Ya., Grigor'eva, L. I., Kramskoi, Ye. D., Korovin, V. B., Kozulya, M. M., Lyssoivan, A. I., Schebetun, A. V., Shapoval, A. N., Shtan', A. F., Solodovchenko, S. I., Voitsenya, V. S., \& Garkusha, I. E. (2014). VHF discharges for wall conditioning at the Uragan-2M torsatron. Nucl. Fusion, 54(3), 033009(5pp). http://dx.doi.org/10.1088/00295515/54/3/033009.

3. Dolgopolov, V. V., \& Stepanov, K. N. (1965). Cerenkov absorption of Alfvén waves and of fast magneto-acoustic waves in an inhomogeneous plasma. Nucl. Fusion, 5(4), 276(12pp). http://dx.doi. org/1088/0029-5515/5/4/003. (in Russian + Eng. transl.).

4. Moiseenko, V. E., Volkov, Ye. D., Tereshin, V. I., \& Stadnik, Yu. S. (2009). Alfvén resonance heating in Uragan-2M torsatron. Plasma Phys. Rep., 35, 828-833.

5. Moiseenko, V. E., Berezhnyj, V. L., Bondarenko, V. N., Burchenko, P. Ya., Castejón, F., Chechkin, V. V., Chernyshenko, V. Ya., Dreval, M. B., Garkusha, I. 
E., Glazunov, G. P., Grigor'eva, L. I., Hartmann, D., Hidalgo, C., Koch, R., Konovalov, V. G., Kotsubanov, V. D., Kramskoi, Ye. D., Kulaga, A. E., Lozin, A. V., Lyssoivan, A. I., Mironov, V. K., Mysiura, I. N., Pavlichenko, R. O., Pashnev, V. K., Romanov, V. S., Shapoval, A. N., Skibenko, A. I., Slavnyi, A. S., Sorokovoy, E. L., Stadnik, Yu. S., Taran, V. S., Tereshin, V. I., \& Voitsenya, V. S. (2011). RF plasma production and heating below ion-cyclotron frequencies in Uragan torsatrons. Nucl. Fusion, 51, 083036. http:// dx.doi.org/10.1088/0029-5515/51/8/083036.

6. Moiseenko, V. E., Kasilov, S. V., Lysojvan, A. I., \& Plyusnin, V. V. (1994). A study of antenna coupling during ICRF plasma build-up. In Proceedings of the 21st EPS Conference Contribution Fusion Plasma Physics, June 27 - July 1, 1994. Montpellier, France. ECA 18B:980-3.

7. Behn, R., Collins, G. A., Lister, J. B., \& Weisen, H. (1987). Observation of density fluctuations at the resonance layers during Alfven wave heating. Plasma Phys. Control. Fusion, 29(1), 75. DOI: 10.1088/07413335/29/1/006.

8. Pashnev, V. K., Sorokovoy, E. L., Berezhnyj, V. L., Burchenko, P. Ya., Volkov, E. D., Krasnyj, V. V., Lozin, A. V., Mironov, Yu. K., Petrushenya, A. A., Pinos, I. B., Skibenko, A. I., Slavnyj, A. S., Dreval, M. B., Kulaga, A. Ye., Tsybenko, S. A., \& Es'kov, A. Yu. (2010). Transition to the improved confinement mode in torsatron
$\mathrm{U}-3 \mathrm{M}$ in range of rare collision frequencies. Probl. Atom. Sci. Techn. Ser. Plasma Phys., 16(6), 17-20.

9. Dreval, M. (2015). First results of multichord soft X-ray detection Aaray on the U-3M Torsatron. Probl. Atom. Sci. Techn. Ser. Plasma Phys., 16(1), 8-11.

10. Chechkin, V. V., Pankratov, I. M., Grigor'eva, L. I., Beletskii, A. A., Kasilov, A. A., Burchenko, P. Ya., Lozin, A. V., Tsybenko, S. A., Slavnyj, A. S., Litvinov, A. P., Kulaga, A. Ye., Pavlichenko, R. O., Zamanov, N. V., Mironov, Yu. K., Romanov, V. S., Pashnev, V. K., Maznichenko, S. M., \& Volkov, Ye. D. (2012). RF discharge dynamics with passing over L- and $\mathrm{H}$-like mode states in the Uragan-3M Torsatron. Probl. Atom. Sci. Techn. Ser. Plasma Phys., 82(6), 3-7.

11. Pankratov, I. M., Beletskii, A. A., Berezhnyj, V. L., Burchenko, P. Ya., Chechkin, V. V., Grigor'eva, L. I., Hartmann, D., Koch, R., Konovalov, V. G., Kulaga, A. Ye., Lesnyakov, G. G., Hartmann, D., Lozin, A. V., Lyssoivan, A. I., Maznichenko, S. M., Mironov, Yu. K., Moiseenko, V. Ye., Pashnev, V. K., Petrushenya, A. A., Prokopenko, A. P., Romanov, V. S., Shapoval, A. N., Shtan', A. F., Sitnikov, D. A., Skibenko, A. I., Slavnyj, A. S., Solodovchenko, S. I., Sorokovoy, E. L., Sorokovoy, Ye. L., Shvets, O. M., Stadnik, Yu. S., Tarasov, I. K., Tereshin, V. I., Tsybenko, S. A., Zamanov, N. V., \& Volkov, Ye. D. (2010). Behavior of RF discharge plasmas in the Uragan-3M and Uragan-2M Torsatrons. Contrib. Plasma Phys., 50(6/7), 520-528. 